\title{
Severa ipocalcemia conseguente all'assunzione orale di citrato in un paziente in emodialisi cronica
}

\author{
Marco Gallo ${ }^{1}$, Stefano Aterini ${ }^{1}$, Sandro Bandini ${ }^{1}$, Franco Bergesio ${ }^{1}$, Anna Maria Ciciani ${ }^{1}$, Lorenzo Aterini ${ }^{1}$, \\ Francesca Calderini², Fiamma Balboni ${ }^{2}$
}

${ }^{1}$ S.O. Nefrologia e Dialisi - Istituto Fiorentino di Cura e Assistenza (IFCA), Firenze - Italy

${ }^{2}$ Laboratorio Analisi - Istituto Fiorentino di Cura e Assistenza (IFCA), Firenze - Italy

\begin{abstract}
Severe hypocalcaemia following oral citrate intake in a patient on chronic hemodialysis
We report a case of an 81-year-old man with end stage renal disease (ESRD) in chronic hemodialysis with severe hypocalcaemia secondary to the intake of a parapharmaceutical containing sodium and potassium citrate and in association with poor dialysis efficiency, due to malfunction of the vascular access, which promptly resolved after discontinuation of the supplement. The patient never showed signs or symptoms of hypocalcaemia. This case highlightens the importance of a correct pharmacological reconciliation in dialysis patients in order to avoid the onset of adverse events due to the uncontrolled intake of drugs or parapharmaceuticals.
\end{abstract}

Keywords: Hemodialysis, Hypocalcaemia, Oral Citrate, Parapharmaceuticals, Vascular Access Failure

\section{Introduzione}

Descriviamo il caso clinico di un paziente anurico in emodialisi cronica in cui l'associazione della scarsa efficienza dialitica per malfunzionamento dell'accesso vascolare con l'assunzione (non nota al nefrologo) di un parafarmaco contenente citrato di sodio e potassio ha determinato una severa ipocalcemia con alterazione dei biomarcatori del metabolismo calcio-fosforo. È noto che il citrato sia un potente chelante del calcio libero circolante, ma gli effetti della sua assunzione, nel paziente con funzione renale altamente compromessa, possono determinare situazioni cliniche inaspettate. Considerata la frequenza con cui il Nefrologo assiste il paziente in dialisi, riveste grande importanza la corretta riconciliazione farmacologica, ripetuta ciclicamente nel tempo, al fine di accertare quali siano i farmaci realmente assunti dal paziente.

\section{Presentazione}

Il caso clinico riguarda un maschio caucasico di 81 anni affetto da cardiopatia ischemica e fibrillazione atriale cronica,

Received: August 3, 2021

Accepted: August 5, 2021

Published online: September 13, 2021

Indirizzo per la corrispondenza:

Marco Gallo

S.O. Nefrologia e Dialisi

Istituto Fiorentino di Cura e Assistenza (IFCA)

Via del Pergolino 4/6

50139 Firenze - Italy

m.gallo@giomi.com in terapia con dicumarolici e digossina e portatore di pacemaker bicamerale, con gammopatia monoclonale IgG- $\lambda$, pregressa infezione da HCV e malattia renale cronica (MRC) da nefroangiosclerosi, in trattamento emodialitico trisettimanale da 2 anni con diuresi residua assente. La prescrizione dialitica prevedeva: bicarbonato dialisi, 240 minuti per seduta, flusso sangue a $300 \mathrm{~mL} / \mathrm{min}$ e filtro dialisi Poracton PAES/PVP 1,8 $\mathrm{m}^{2}$ (Revaclear $400^{\circ}$ ). Le concentrazioni ioniche del dialisato erano le seguenti: Potassio $\left(\mathrm{K}^{+}\right): 3 \mathrm{mEq} / \mathrm{L}$; Sodio $\left(\mathrm{Na}^{+}\right)$: $142 \mathrm{mEq} / \mathrm{L}$; Calcio $\left(\mathrm{Ca}^{++}\right): 1,5 \mathrm{mmol} / \mathrm{L}$; Magnesio $\left(\mathrm{Mg}^{++}\right): 0,5 \mathrm{mg} / \mathrm{dL}$; Cloro $\left(\mathrm{Cl}^{-}\right)$: 146,5 mEq/L; Bicarbonato ( $\left.\mathrm{HCO3}^{-}\right)$: $31 \mathrm{mmol} / \mathrm{L}$.

Lo studio del metabolismo minerale del paziente aveva evidenziato, nei 6 mesi precedenti, un iperparatiroidismo secondario di grado lieve (Paratormone [PTH]: $205 \mathrm{ng} / \mathrm{L}$; Calcemia totale $\left[\mathrm{tCa}^{++}\right.$]: $8,9 \mathrm{mg} / \mathrm{dL}$; Calcio ionizzato [iCa ${ }^{++}$: $4,83 \mathrm{mg} / \mathrm{dL}$; Fosforo $[\mathrm{P}]: 4,5 \mathrm{mg} / \mathrm{dL}$ ), senza necessità di terapie con Vitamina D (o analoghi), Calciomimetici, Chelanti dei fosfati o supplementi di Calcio (Fig. 1).

Nel corso degli ultimi 3 mesi, il paziente lamentava disappetenza, nausea ed episodi di vomito post-prandiale, che gli impedivano un corretto apporto alimentare, evidenziato anche dal rilievo di una costante ipofosforemia ( $P: 1,5 \mathrm{mg} / \mathrm{dL}$ ). Veniva eseguita l'esofagogastroduodenoscopia, che rilevava un'ernia iatale e un'iperemia diffusa della mucosa gastrica (alla biopsia: lieve iperplasia foveolare e delle cellule parietali), ma, nonostante la terapia con inibitori di pompa protonica (PPI), il paziente continuava a lamentare la persistenza della medesima sintomatologia.

Il paziente era portatore di fistola artero-venosa (FAV) per emodialisi omero-basilica prossimale, che, per la presenza di recidive stenotiche, era stata sottoposta negli ultimi 4 mesi a ripetute angioplastiche. Sette giorni prima dell'evento 


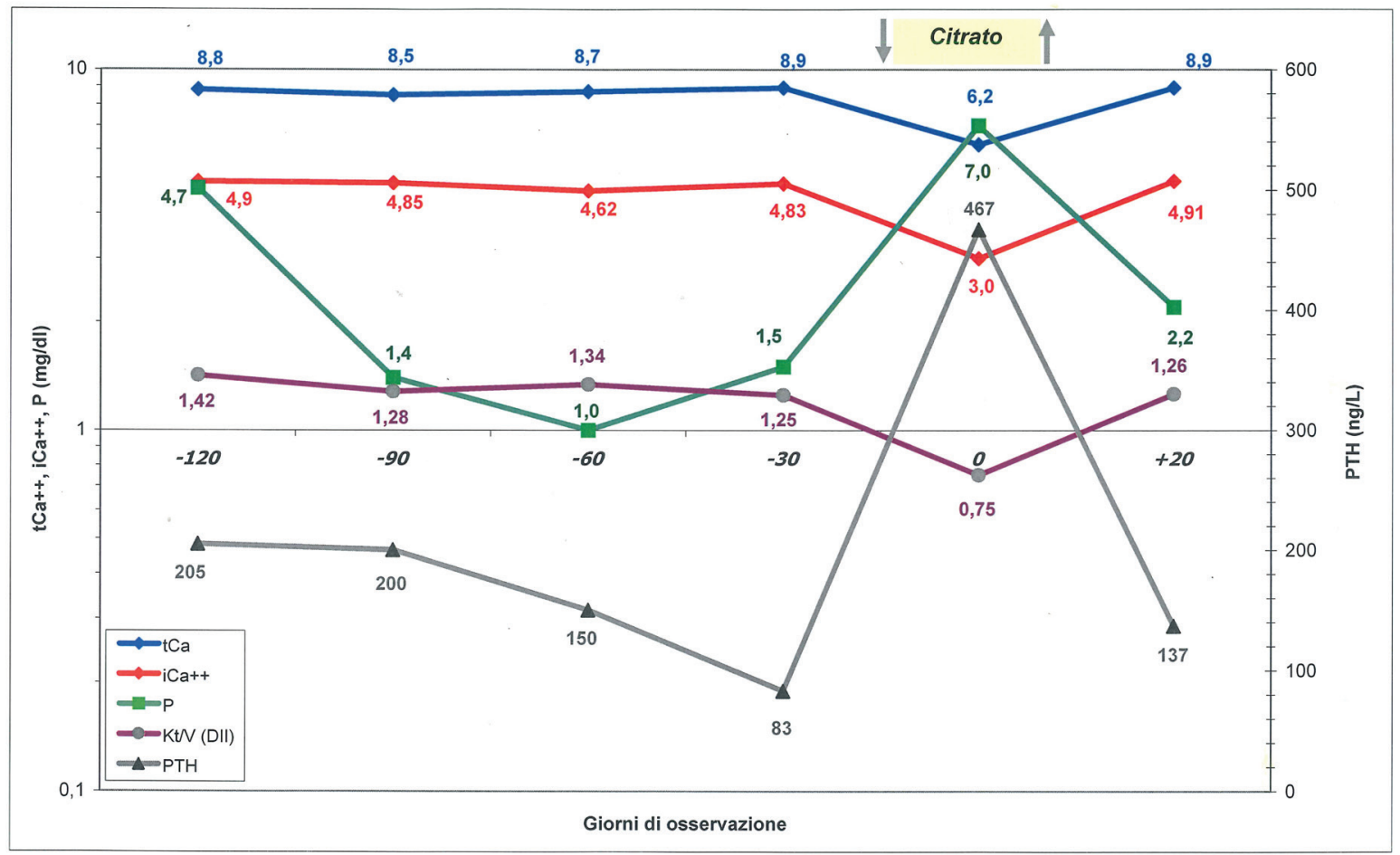

Fig. 1 - Andamento temporale dei valori sierici predialitici di calcemia totale $\left(\mathrm{tCa}^{++}\right)$, calcio ionizzato (iCa $\left.{ }^{++}\right)$, fosforo $(\mathrm{P})$, paratormone (PTH) e del Kt/V (sec. Daugirdas II) nei 120 giorni precedenti e nei 20 giorni successivi al riscontro di ipocalcemia. L'inizio e l'interruzione dell'assunzione del citrato sono contrassegnati, rispettivamente, dalle frecce verso il basso e verso l'alto. In concomitanza con la scarsa efficienza dialitica (Kt/V: 0,75$)$ al giorno " 0 " si è manifestata la grave ipocalcemia con incremento consensuale di P e PTH. Dopo la sospensione del citrato e il ripristino dell'efficienza dialitica si è assistito alla correzione dei parametri ematochimici in linea con i valori precedenti l'assunzione dell'integratore.

descritto in questo caso clinico si osservava, al termine della seduta emodialitica, la comparsa di trombosi parziale della vena basilica (nonostante la terapia con Warfarin), sottoposta 24 ore dopo a una disostruzione con ripristino parziale della funzionalità dell'accesso vascolare. Le indagini effettuate immediatamente prima dell'evento trombotico mostravano un'elevata percentuale di ricircolo ( $>25 \%$ al test dell'Urea) e una bassa portata della FAV (media di 3 misurazioni effettuate sull'arteria omerale: $<500 \mathrm{~mL} / \mathrm{min}$ ), con conseguente riduzione dell'efficienza dialitica. Gli esami ematici, prelevati in occasione dei controlli mensili di routine (7 giorni dopo), rivelavano un dato completamente nuovo: la presenza di una marcata ipocalcemia pre-dialitica $\left(\mathrm{tCa}^{++}: 6,2 \mathrm{mg} / \mathrm{dL}\right.$, Calcemia corretta perl'Albumina: $6,4 \mathrm{mg} / \mathrm{dL}$ ) con un improvviso aumento del Fosforo (7,0 mg/dL) e del Paratormone (PTH: $467 \mathrm{ng} / \mathrm{L})$. Anche la misurazione della calcemia ionizzata, effettuata su 2 differenti strumenti per emogasanalisi (EGA), confermava la severa ipocalcemia (iCa ${ }^{++}: 3,0 \mathrm{mg} / \mathrm{dL}$ ). L'EGA prelevata a fine trattamento emodialitico mostrava, però, una completa correzione dell'ipocalcemia (iCa ${ }^{++}: 5,06 \mathrm{mg} / \mathrm{dL}$ ), a fronte di una concentrazione di calcio nel dialisato pari a $1,5 \mathrm{mmol} / \mathrm{L}$ $(6,0 \mathrm{mg} / \mathrm{dL})$. Al trattamento dialitico successivo erano stati valutati nuovamente tutti i parametri ematochimici, che confermavano l'ipocalcemia pre-dialitica $\left(\mathrm{tCa}^{++}: 6,2 \mathrm{mg} / \mathrm{dL}\right.$;
Calcemia corretta per l'Albumina: 6,4 mg/dL; iCa ${ }^{++}: 3,1 \mathrm{mg} / \mathrm{dL}$; $\mathrm{tCa}^{++} / \mathrm{iCa}^{++}$ratio: 2,0; $\mathrm{PTH}: 450 \mathrm{ng} / \mathrm{L} \mathrm{Mg}^{++}$: $1,7 \mathrm{mg} / \mathrm{dL} ; \mathrm{K}^{+}$: $3,4 \mathrm{mEq} / \mathrm{L} ; \mathrm{P}: 6,3 \mathrm{mg} / \mathrm{dL}$ ) e una calcemia ionizzata postdialitica al di sopra del valore atteso ( $\mathrm{iCa}^{++}: 6,5 \mathrm{mg} / \mathrm{dL}$ ) (Tab. I).

Veniva, quindi, effettuata una ricognizione farmacologica al fine di valutare se i farmaci da noi prescritti fossero realmente assunti e, nel corso dell'intervista, emergeva che il paziente, 15 giorni prima, si era recato privatamente da un Gastroenterologo che, per le sue problematiche dispeptiche, aveva prescritto un integratore che era stato regolarmente assunto e la cui scheda tecnica riportava la presenza (nella dose abituale di 40 gocce) di Vitamina B1 (0,9 mg), Vitamina B2 (1,35 mg), Vitamina B6 (0,9 mg), L-Alanina (7,5 mg), Zenzero estratto idroalcolico $(12,5 \mathrm{mg})$, Sodio citrato $(150 \mathrm{mg})$ e Potassio citrato $(127,5 \mathrm{mg})$. L'integratore era stato immediatamente sospeso e, contestualmente al miglioramento dell'efficienza dialitica per chiusura della FAV e posizionamento di catetere venoso centrale permanente, si assisteva, dopo pochi giorni, alla completa normalizzazione dei parametri ematochimici (Tab. I), senza necessità di farmaci per il controllo del PTH o dell'iperfosforemia. Ai controlli di routine del mese successivo, si evidenziava, infatti, un quadro in linea con i parametri ematici prima dell'assunzione dell'integratore (Calcemia: 8,9 mg/dL; iCa++: 4,91 mg/dL; P: 2,2 mg/dL; PTH: 137 ng/L). 
TABELLA I - Parametri ematochimici pre- e post-dialitici nei 4 mesi precedenti e nei 20 giorni successivi al riscontro di ipocalcemia.

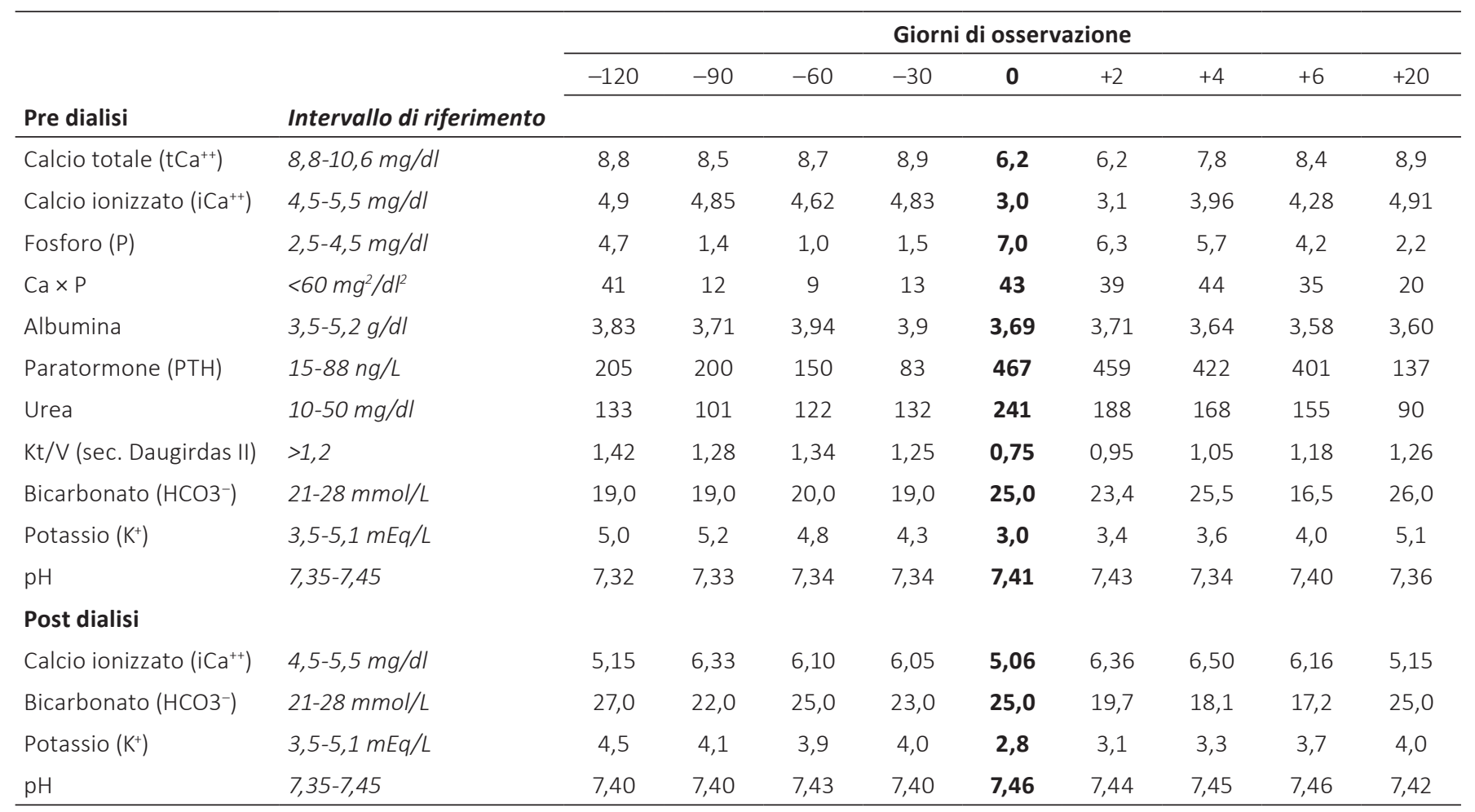

\section{Discussione}

Il citrato è un composto organico con peso molecolare di 189 Dalton, coinvolto nel ciclo dell'acido tricarbossilico, nella regolazione dell'equilibrio acido-base, nel metabolismo dei lipidi e nella formazione dell'osso. In quanto metabolita intermedio del ciclo di Krebs, rappresenta una fonte energetica che contribuisce in modo significativo al metabolismo ossidativo del rene e del fegato (1). II citrato plasmatico viene interamente filtrato dal glomerulo e parzialmente riassorbito a livello del tubulo prossimale e ha un'escrezione frazionale variabile dal $10 \%$ al $40 \%$ (2). II basso peso molecolare del citrato e il quasi assente legame con le proteine plasmatiche spiegano una filtrazione glomerulare pari al $100 \%$ e un'elevata rimozione tramite l'emodialisi. Nel sangue, circola, però, solo una piccola quota del citrato corporeo, in quanto il $90 \%$ è depositato nel tessuto osseo, che rappresenta, perciò, l'organo dove viene conservato (3).

Il citrato forma complessi molto stabili con il calcio, riducendo il calcio libero e rendendolo meno disponibile alla formazione di sali poco solubili (4). Dal momento che il calcio ionizzato è un fattore essenziale per la coagulazione, l'aggregazione piastrinica, l'attivazione dei leucociti e l'attivazione della via alterna del complemento, l'anticoagulazione regionale con citrato è frequentemente utilizzata nell'emodialisi cronica e nelle tecniche sostitutive renali in area critica (5).

La sua spiccata affinità per il calcio con formazione di complessi solubili e la sua funzione di interferenza negativa con i processi di cristallizzazione dei sali di calcio rendono questa molecola estremamente importante nella fisiopatologia della calcolosi urinaria. II citrato urinario, infatti, è efficace nella prevenzione delle recidive di calcolosi perché inibisce l'aggregazione e la crescita dei cristalli di ossalato di calcio e di fosfato di calcio (6), come dimostrato da diversi trial randomizzati (7). L'ipocitraturia è, infatti, una frequente condizione che si riscontra nella nefrolitiasi e che può essere corretta dalla somministrazione di sali alcalini (usualmente 3-9 g/die di citrato di potassio e/o di magnesio suddivisi in 2-3 somministrazioni giornaliere). Nel lume intestinale, il citrato forma complessi solubili con i principali cationi e viene assorbito con un sistema di trasporto analogo a quello renale.

Solitamente, però, il citrato di potassio, terapia cardine della nefrolitiasi, non è mai utilizzato, per ovvie ragioni, nel paziente con funzione renale altamente compromessa come nel dializzato. Pertanto, non sono note quali siano le conseguenze di croniche assunzioni di citrato per via orale nel paziente anurico in emodialisi in cui si associ una scarsa efficienza dialitica.

Nel nostro caso, l'elemento nodale della successione degli eventi sembra essere, considerata la notevole rimozione dialitica del citrato, proprio il malfunzionamento dell'accesso vascolare. Infatti, l'elevata percentuale di ricircolo della FAV, associata alla contemporanea assunzione di citrato di sodio e potassio presente nell'integratore, aveva determinato il progressivo accumulo del citrato plasmatico e la conseguente ipocalcemia. Al termine delle sedute dialitiche, si assisteva a una correzione dell'ipocalcemia per rimozione del citrato da parte del filtro dialisi e liberazione di calcio ionizzato dallo 
stesso, che, associato a quello acquisito dal bagno dialisi per diffusione, determinava livelli normalizzati di $\mathrm{iCa}^{++}$. Ma tale correzione è stata, probabilmente, di breve durata per una rimozione solo parziale del citrato plasmatico a causa del malfunzionamento della FAV. Benché sia descritto che la tossicità da citrato si evidenzia solo quando il iCa ${ }^{++}$si riduce a valori $<0,9 \mathrm{mmol} / \mathrm{L}(3,6 \mathrm{mg} / \mathrm{dL})(8)$, il paziente non ha mai manifestato segni o sintomi di ipocalcemia.

L'incremento dei livelli di PTH (fino a circa 6 volte rispetto ai valori precedenti) ben si concilia con la grave ipocalcemia; le paratiroidi sono, infatti, in grado di rilevare variazioni minime della concentrazione del calcio ionizzato mediante i recettori sensibili al calcio (CaSR) localizzati sulla membrana delle cellule paratiroidee, regolando, così, "minute to minute" il rilascio del PTH (9). II PTH secreto stimola il riassorbimento osseo mediante meccanismi complessi (osteolisi osteoclastica), che comportano la liberazione di calcio e fosforo inorganico nei liquidi extracellulari al fine di mantenere costante il prodotto calcio-fosforico. II tempo relativamente breve degli eventi e, soprattutto, l'accumulo di citrato plasmatico non hanno permesso di rilevare incrementi della calcemia ionizzata.

L'incremento dei livelli del fosforo $(7,0 \mathrm{mg} / \mathrm{dL})$ è stato notevole, considerando le fosforemie precedenti (1,4$1,5 \mathrm{mg} / \mathrm{dL}$ ). L'iperfosforemia, come già descritto, si potrebbe attribuire all'azione del PTH sull'osso che, oltre al calcio, riassorbe anche il fosforo. Ma anche l'assunzione, anche se di modeste dosi giornaliere, di fosforo contenuto nell'estratto idroalcolico dello zenzero presente nell'integratore (168 mg/100 g) e l'inefficienza della dialisi potrebbero avere avuto un ruolo complementare.

L'assenza di rilievo dell'acidosi metabolica, prevedibile in un nefropatico cronico in terapia sostitutiva con ridotta efficienza dialitica, è un'ulteriore conferma del ruolo svolto dal citrato. II metabolismo del citrato si traduce in un netto aumento dell'apporto di bicarbonato e il riscontro di ipopotassiemia $\left(\mathrm{K}^{+}: 3,0\right.$ $\mathrm{mEq} / \mathrm{L}$ ) potrebbe, verosimilmente, essere giustificato dall'alcalosi metabolica indotta dall'effetto alcalinizzante del citrato. È ben noto, infatti, che la terapia alcalinizzante, correggendo l'acidosi metabolica, contribuisce ad abbassare la kaliemia, favorendo lo spostamento intracellulare del potassio.

Un dato è, comunque, certo: con il ripristino della funzionalità dell'accesso vascolare e con la sospensione dell'integratore, ai controlli ematici successivi si evidenziavano, in tempi relativamente brevi, parametri ematochimici assimilabili a quelli rilevati prima dell'assunzione dell'integratore (Tab. I). Proprio in virtù della rapidità con cui si sono svolti gli eventi non si è reso necessario modificare né la concentrazione del calcio ione nel bagno di dialisi né la terapia del paziente, che non ha assunto farmaci per il controllo degli elevati livelli sierici di PTH e dell'iperfosforemia.

Pur con le limitazioni che questo caso clinico presenta, è interessante notare come l'associazione tra l'assunzione orale di citrato e la scarsa efficienza dialitica possa aver determinato una combinazione di eventi che hanno portato a evidenziare quanto descritto. Rimane, tuttavia, di grande importanza l'accurata indagine sui farmaci assunti dai pazienti in dialisi e sulla corretta riconciliazione farmacologica ripetuta ciclicamente nel tempo. Frequentemente, infatti, vengono prescritti (anche da altri specialisti e, a volte, all'insaputa del Nefrologo curante) o assunti autonomamente dal paziente integratori alimentari o complessi vitaminici, la cui corretta identificazione potrebbe consentire di giustificare l'interferenza farmacologica e l'alterazione di parametri ematochimici altrimenti non spiegabili, come, per esempio, l'ipocalcemia descritta nel nostro caso o, come in altre circostanze, l'iperpotassiemia e I'iperfosforemia, che costituiscono, per il Nefrologo, una sfida quotidiana e costante.

\section{Acknowledgements}

Si ringraziano tutti gli Infermieri del Centro Dialisi Ulivella per l'accuratezza, la dedizione e la professionalità con cui prestano la propria opera.

\section{Disclosures}

Conflict of interest: The authors declare no conflict of interest. Financial support: This research received no specific grant from any funding agency in the public, commercial, or not-for-profit sectors.

\section{Bibliografia}

1. Simpson DP. Citrate excretion: a window on renal metabolism. Am J Physiol. 1983;244(3):F223-F234. PubMed

2. Hamm LL. Renal handling of citrate. Kidney Int. 1990;38(4):728 735. CrossRef PubMed

3. Costello LC, Franklin RB. Plasma citrate homeostasis: How it is regulated; and its physiological and clinical implications. An important, but neglected, relationship in medicine. HSOA J Hum Endocrinol. 2016;1:005. PubMed

4. Tiselius HG, Berg C, Fornander AM, Nilsson MA. Effects of citrate on the different phases of calcium oxalate crystallization. Scanning Microsc. 1993;7(1):381-389. PubMed

5. Seaton RD, Duncan KA, Pinnick RV, Diederich DA, Wiegmann TB. Regional citrate anticoagulation in chronic hemodialysis patients. Trans Am Soc Artif Intern Organs. 1983;29:414418. PubMed

6. Wolfgram DF, Gundu V, Astor BC, Jhagroo RA. Hydrochlorothiazide compared to chlorthalidone in reduction of urinary calcium in patients with kidney stones. Urolithiasis. 2013;41(4):315-322. CrossRef PubMed

7. Phillips R, Hanchanale VS, Myatt A, Somani B, Nabi G, Biyani CS. Citrate salts for preventing and treating calcium containing kidney stones in adults. Cochrane Database Syst Rev. 2015;(10):CD010057. CrossRef PubMed

8. Monchi M. Citrate pathophysiology and metabolism. Transfus Apheresis Sci. 2017;56(1):28-30. CrossRef PubMed

9. Olgaard K, Salusky IB, Silver J. The spectrum of mineral bone disorders in chronic kidney disease. 2nd ed. Oxford. Oxford University Press; 2010:111-114. CrossRef 